\title{
A Case of Generalized Auditory Agnosia with Unilateral Subcortical Brain Lesion
}

\author{
Hyee Suh, M.D., Yong-Il Shin, M.D., Soo Yeon Kim, M.D., Sook Hee Kim, Ph.D. , Jae Hyeok Chang, M.D., \\ Yong Beom Shin, M.D., Hyun-Yoon Ko, M.D.
}

Department of Rehabilitation Medicine, Pusan National University School of Medicine, Research Institute for Convergence of Biomedical Science and Technology, Pusan National University Yangsan Hospital, Yangsan 626-770,

${ }^{1}$ Department of Speech \& Language Therapy, Young Dong University, Chungbuk 370-701, Korea

The mechanisms and functional anatomy underlying the early stages of speech perception are still not well understood. Auditory agnosia is a deficit of auditory object processing defined as a disability to recognize spoken languages and/or nonverbal environmental sounds and music despite adequate hearing while spontaneous speech, reading and writing are preserved. Usually, either the bilateral or unilateral temporal lobe, especially the transverse gyral lesions, are responsible for auditory agnosia. Subcortical lesions without cortical damage rarely causes auditory agnosia. We present a 73-year-old right-handed male with generalized auditory agnosia caused by a unilateral subcortical lesion. He was not able to repeat or dictate but to perform fluent and comprehensible speech. He could understand and read written words and phrases. His auditory brainstem evoked potential and audiometry were intact. This case suggested that the subcortical lesion involving unilateral acoustic radiation could cause generalized auditory agnosia.

Key Words Auditory agnosia, Unilateral subcortical lesion

\section{INTRODUCTION}

Auditory agnosia is a rare otoneurological manifestation that refers to the defective recognition of auditory stimuli in the context of preserved hearing and is charac-

Received July 26, 2011; Accepted December 28, 2011

Corresponding author: Yong-Il Shin

Department of Rehabilitation Medicine, Pusan National University School of Medicine, Research Institute for Convergence of Biomedical Science and Technology, Pusan National University Yangsan Hospital, 20, Geumo-ro, Mulgeum-eup, Yangsan 626-770, Korea

Tel: +82-55-360-2872, Fax: +82-55-360-2169, E-mail: rmshin01@gmail. com

(c) This is an open-access article distributed under the terms of the Creative Commons Attribution Non-Commercial License (http:// creativecommons.org/licenses/by-nc/3.0) which permits unrestricted noncommercial use, distribution, and reproduction in any medium, provided the original work is properly cited.

Copyright $\odot 2012$ by Korean Academy of Rehabilitation Medicine terized by preserved functioning with language such as reading, writing and speaking. ${ }^{1}$

There are three different types of auditory agnosia. Verbal auditory agnosia or pure word deafness has a selective decrease in the recognition of verbal sounds. And non-verbal auditory agnosia has a selective decrease in the recognition of non-verbal sounds. A generalized decrease in both verbal and non-verbal sounds is generalized auditory agnosia. These symptoms substantially overlap and can be difficult to separate from each other. ${ }^{2}$ Usually either the bilateral or unilateral temporal lobe especially the supratemporal gyrus, Heschl's gyrus, known as the primary auditory cortex are responsible for auditory agnosia. ${ }^{3}$ And generalized auditory agnosia is caused by the primary auditory cortex and auditory association cortex. ${ }^{2}$ Subcortical lesions without cortical damage rare- 
ly causes auditory agnosia. Here, we report a generalized auditory agnosia patient caused by unilateral subcortical lesion with intracranial hemorrhage (ICH) in temporal area.

\section{Case Report}

A 73-year-old right handed male presented to emergency room with the sudden onset of right hemiplegia and dysarthria. He was diagnosed as having intracranial hemorrhage of the left basal ganglia and temporal lobe. After 3 weeks, he was offered rehabilitation services for the auditory problem of being unable to differentiate voices, music, and environmental sounds. His past medical history included hypertension and right basal ganglia intracranial hemorrhage 13 years previous. There has been no definite sequelae of previous stroke. In the neuroimaging studies 2 weeks after the onset of the stroke, a non-contrast computed tomography (CT) scan and magnetic resonance imaging (MRI) showed intracranial hemorrhage of the left basal ganglia and temporal lobe area and a single photon emission computed tomography (SPECT) showed a moderate to severe perfusion decrease and impaired vascular reserve in left temporal lobe (Fig. 1).

He was an alert patient but, he did not follow oral commands or respond to environmental sounds. His verbal repetition and writing to dictation were severely defective with phonemic substitutions. Neuropsychological evaluations were performed 3 weeks after the onset of stroke.
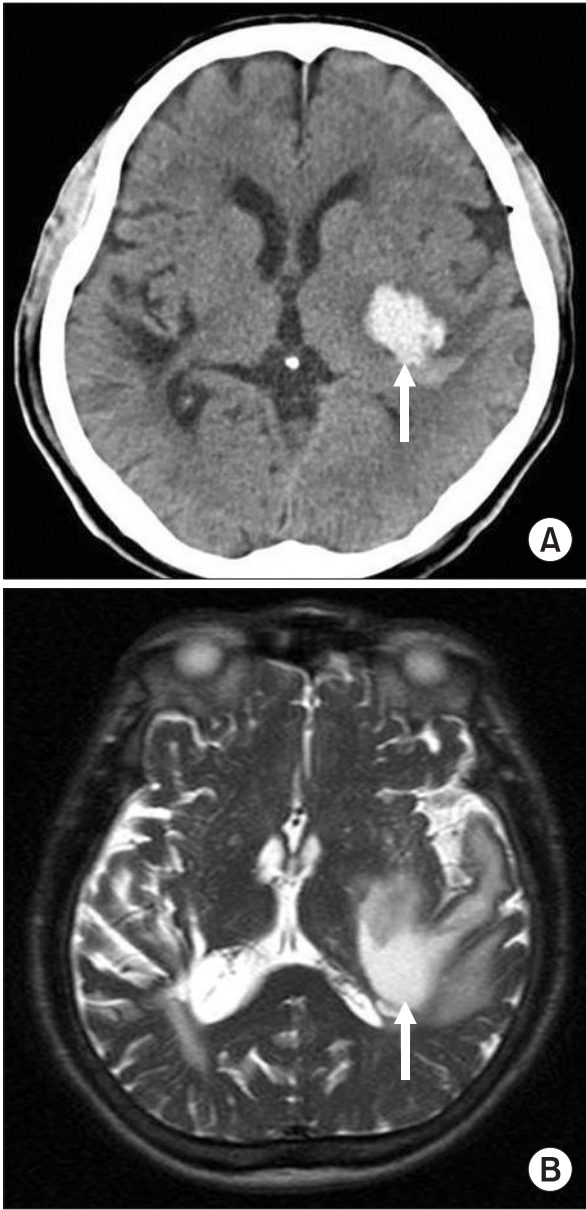
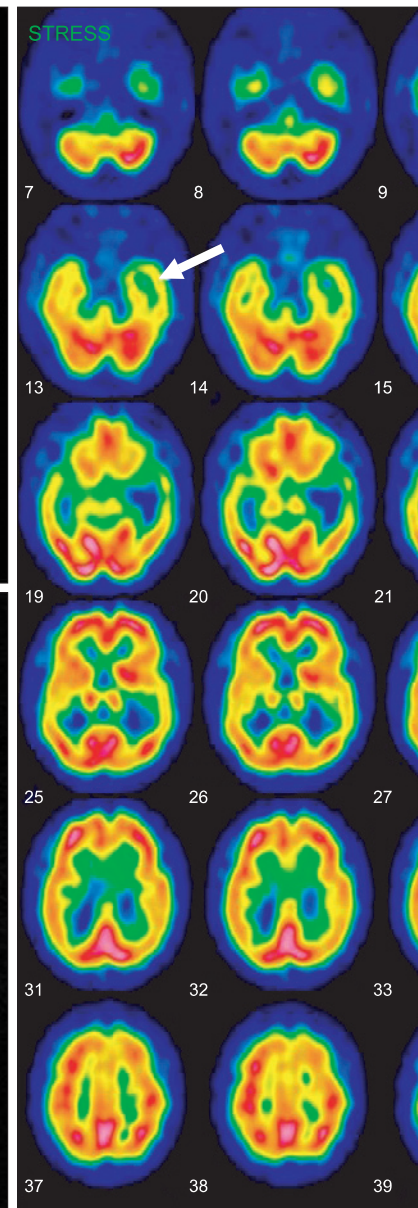

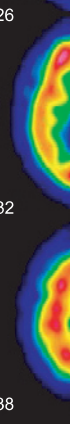

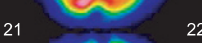

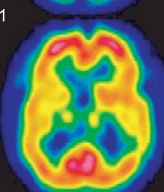

27

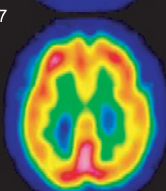

33

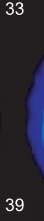

40
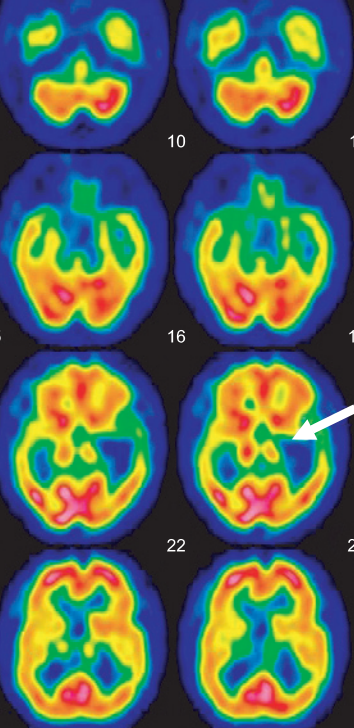

28

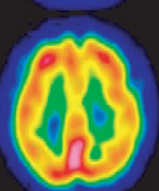

34
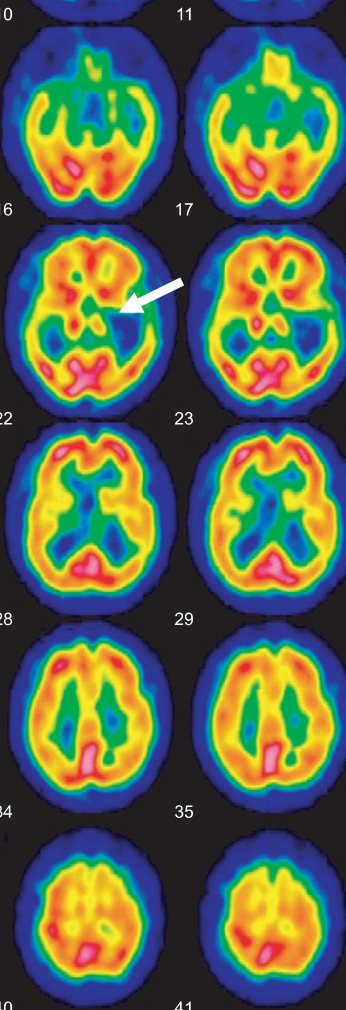
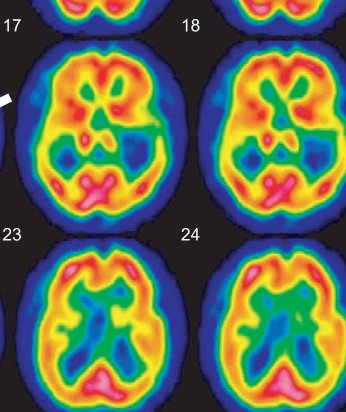

29
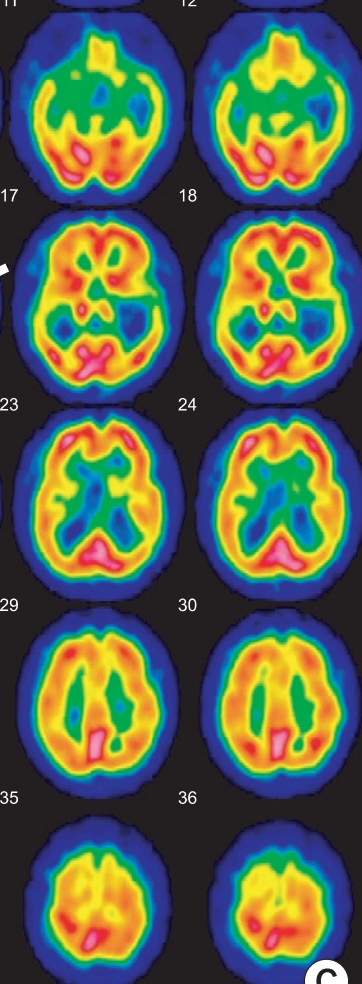

41

Fig. 1. (A) A computed tomography image that shows acute intracranial hemorrhage at the left striatocapsular area with mild perihematoma edema. (B) The MRI T2-weighted axial images showed a large subacute hematoma in the left basal ganglia, internal capsule and temporal white matter with mild mass effect. The cerebral white matter including left auditory radiation was severely damaged, whereas the auditory cortexes were intact. (C) The brain SECT image showed a severe perfusion decrease and an impaired vascular reserve in the left temporal lobe. Also a moderate perfusion decrease and preserved vascular reserve in the left basal ganglia were shown. 


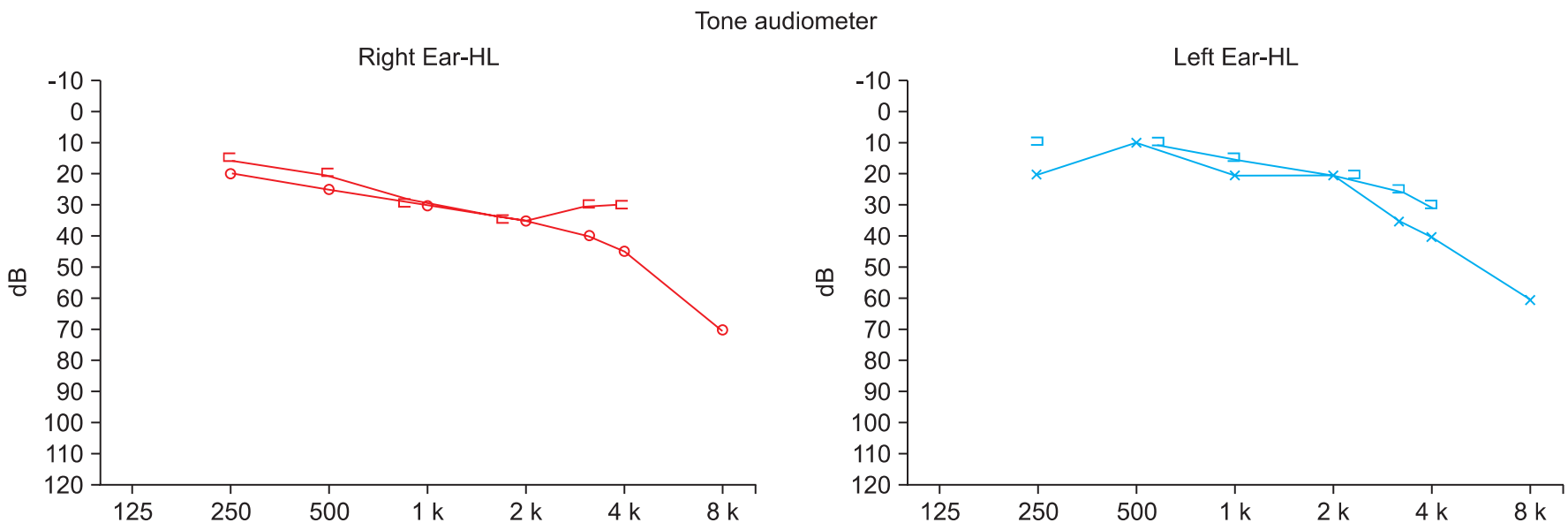

Fig. 2. Pure tone audiometry that showed nearly normal bilateral findings that were considered for his age.

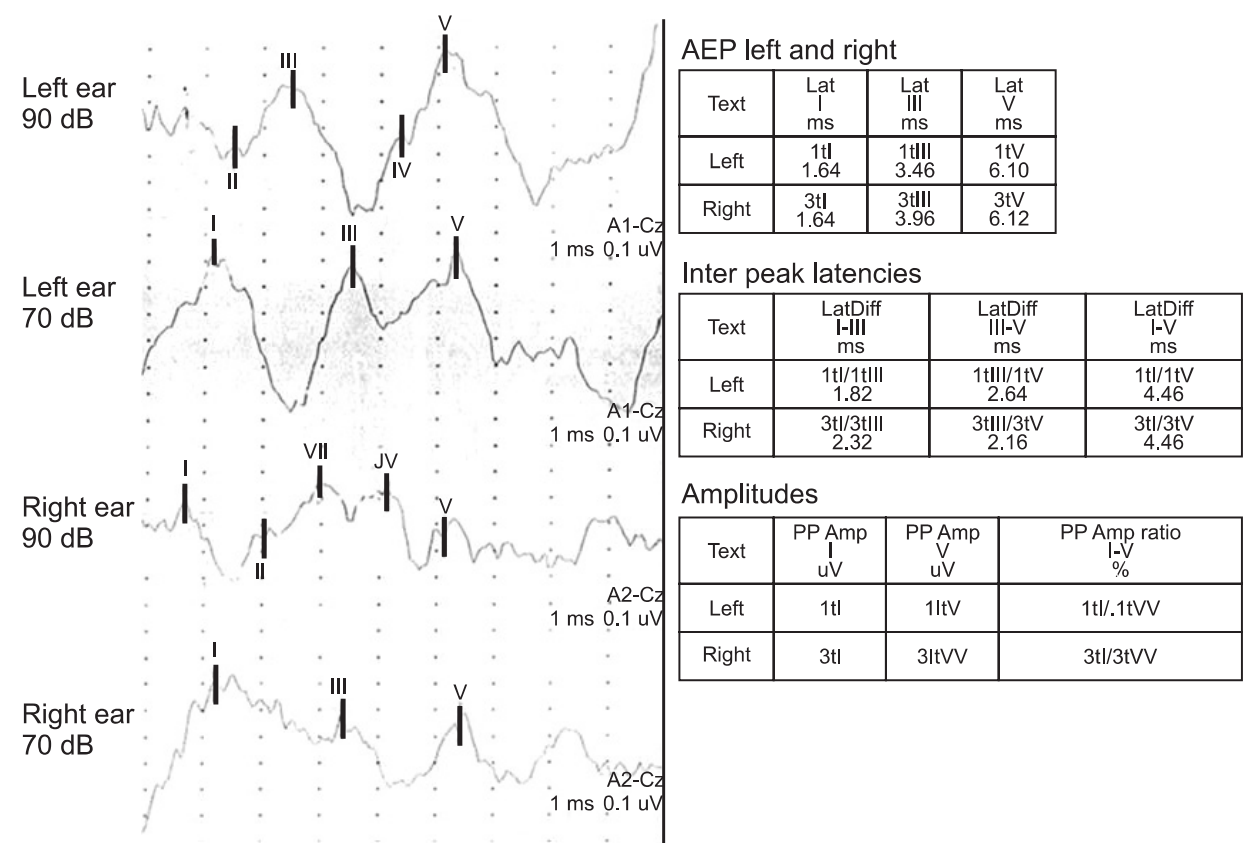

Fig. 3. Brainstem auditory-evoked potentials (BAEP) of the patient 3 weeks after stroke onset showed normal bilateral latencies and amplitudes.
He obtained a score of $4 / 30$ on the Mini Mental State Exam of Korea (MMSE-K) with verbal communication. He was diagnosed as having cognitive dysfunction. But he could comprehend written and gestural communication. A language assessment using the Korean Version of the Western Aphasia Battery (K-WAB) revealed that he had significant deficits in auditory comprehension, repetition, and writing to dictation, despite the presence of good reading, fluent speech, and spontaneous writing, without evidence of aphasia. He could speak complete sentences without paraphasia. The Token test was $0 \%$ for verbal communication but $20.9 \%$ (13 items out of 62 ) for written communication. He came close to be misdiagnosed as Wernicke's aphasia but, his language disorder was different from Wernicke's aphasia for naming, reading and spontaneous writing. He obtained a score of $21 / 30$ on MMSE-K with written communication. Therefore, he was regarded as not having severe cognitive dysfunction for his age and educational status. He could not differentiate people's voices, music, and environmental sounds. Everything sounded like a disagreeable noise, a "buzzing", including pure tone signals. Standard pure tone threshold audiometry was conducted. The standard pure tone threshold audiometry showed normal findings (Fig. 2). Also brainstem auditory-evoked potentials (BAEP) exhibited normal bilateral latencies (Fig. 3). The visuo-cognitive and visuo-spatial functions were preserved. The results of evaluation studies are summarized 
Table 1. Summary of Evaluation Studies

\begin{tabular}{lcc}
\hline \multirow{2}{*}{ Examination } & \multicolumn{2}{c}{ Findings } \\
\cline { 2 - 3 } & $\begin{array}{c}\text { Verbal } \\
\text { communication }\end{array}$ & $\begin{array}{c}\text { Written } \\
\text { communication }\end{array}$ \\
\hline MMSE-K & $4 / 30$ & $21 / 30$ \\
Token test & $0 \%$ & $20.9 \%$ \\
Tone audiometry & \multicolumn{2}{c}{ Normal } \\
BAEP & \multicolumn{3}{c}{ Normal } \\
\hline
\end{tabular}

in Table 1.

He could hear sounds but had difficulty in identifying words and environmental sounds such as: male and female voices, music, a baby crying, laughing sounds, an animal crying (cat, dog, cow, horse, pig, bird), instrumental sounds (piano, clocks, telephone ring), wind, a wave, and honk. Therefore, he was diagnosed as having generalized auditory agnosia with intracranial hemorrhage in the left basal ganglia and temporal lobe. He had speech therapy for variable sound differentiation and lipreading with a listening program to foster communication skills. Six months after the recent stroke, a follow-up $\mathrm{K}-\mathrm{WAB}$ and audiometry were performed and there was no significant interval improvement with the previous exam. Gradually he regained the ability to communicate and understand almost all the words used in daily life by trained lip-reading.

\section{DISCUSSION}

To our knowledge, auditory agnosia is caused by cerebrovascular diseases such as: a stroke, viral infection of central nervous system (CNS), head trauma, brain tumor, hydrocephalus and variable CNS lesions. The most common cause is cerebrovascular disease. ${ }^{4}$ Clinically auditory agnosia is easily misdiagnosed as cognitive dysfunction, aphasia or deafness. But, it can be differentiated from cognitive dysfunction through a better score with written communication as compared to verbal communication in a cognition evaluation test. Reading, writing and spontaneous speech are possible in auditory agnosia but, are not possible for Wernicke's aphasia. Also repetition is not possible for auditory agnosia unlikely due to transcortical sensory aphasia. It can be differentiated from deafness by audiometry findings. This patient was misdiagnosed as having cognitive dysfunction with poor oral comprehen- sion and a low score in the MMSE-K at the initial evaluation. But, he had a better function in written communication as compared to verbal communication.

Although auditory agnosia typically results from bilateral superior temporal lobe damage, it has also been reported following unilateral damage to the primary auditory cortex. ${ }^{3}$ Non-verbal auditory agnosia has a tendency to be caused by a right unilateral temporal convexity lesion. ${ }^{5}$ Although bilateral primary auditory cortex lesions have been known as the main cause of auditory agnosia, subcortical lesions also could be possible causes.

The most common explanation for the existence of auditory agnosia following a unilateral subcortical lesion is that auditory agnosia can result when preserved language areas of the left hemipshere are unable to receive auditory input from either hemisphere. Unilateral subcortical auditory agnosia has been thought of as a disconnection syndrome of damage to cross-hemispheric pathways through corpus callosal commissural fiber. But, a recent study using diffuse tensor imaging (DTI) suggests that auditory agnosia can arise from a unilateral lesion even in the presence of intact cross-hemispheric connectivity. ${ }^{6}$

This patient had a history of right basal ganglia ICH 13 years previous but a significant sequelae of a previous stroke and an auditory or speech problem, had not remained. So, after we excluded the possibility of a right hemispheric lesion, we diagnosed the manifestation due to acute ICH on the left subcortical lesion. However, it could be possible that since there was previous right unilateral auditory pathway damage, the auditory agnosia that happened with the bilateral auditory pathways were finally interrupted. There are some case reports about auditory agnosia in a unilateral brain lesion with previous brain lesion on the opposite side. ${ }^{7}$ Our patient possibly developed auditory agnosia with bilateral brain lesions consequentially. The brain MRI and SPECT were performed in this patient but, DTI was not performed. Therefore, we couldn't identify the damage of crosshemispheric connectivity.

There are two auditory pathways in the human brain, the primary (specific) auditory pathway and the secondary (nonspecific) auditory pathway. ${ }^{8}$ The primary auditory pathway is involved in auditory perception, whereas the secondary auditory pathway helps maintain arousal and nonspecific auditory perception. Most of the patients with auditory agnosia were reported to have poor audi- 
tory perception but with somewhat preserved hearing. Probably, the secondary auditory pathway contributes to hearing but is not specific to auditory perception. The primary auditory pathway has been studied thoroughly, however, much remains unknown about the secondary auditory pathway and its relationship with the primary pathway.

The explanation for generalized auditory agnosia is that there is damage of the primary auditory pathway that includes the secondary auditory pathway. In our patient, both the primary and secondary auditory pathways may have been damaged. Diffuse damage of the topographic arranged auditory radiations from the medial geniculate body to the temporal lobe can block the pathways in the primary auditory cortex and the auditory association cortex. $^{2}$

In previous study, the left hemisphere is specialized for a rapid temporal processing of complex sounds whereas the right hemisphere is specialized for the processing of dynamic pitch information. ${ }^{3}$

In past studies, musical sounds have been thought of as perceived by the primary auditory pathway but recent studies report that the secondary pathway might have played a role subconsciously in the right hemisphere rather than the left. ${ }^{4,9}$ Perception training with familiar musical sounds for the patient and variable environmental sounds could be effective in the enhancement of non-verbal sound recognition. ${ }^{4}$ This training method proposes that musical and rhythmic sounds can be used as a treatement method for auditory agnosia in a preserved secondary auditory pathway despite the damaged primary auditory pathway patient. Secondary auditory pathway preserved patients with "lip-reading + listening" training can attain a better recovery than those with "lipreading" only. ${ }^{9}$ We can expect that patients with auditory agnosia with a preserved secondary auditory pathway can achieve better rehabilitation outcomes than those without.

The clinical diagnosis of auditory agnosia can be missed or misdiagnosed as other disorders such as sensory aphasia. Close observation and variable evaluation tools of cognition, speech and auditory function are important in order to make an exact diagnosis and treatment of auditory agnosia in stroke patients.

\section{ACKNOWLEDGEMENTS}

This study was supported by a 2011 research grant from Pusan National University Yangsan Hospital.

\section{REFERENCES}

1. Simons JS, Lambon Raiph MA. The auditory agnosias. Neurocase 1999; 5: 379-406

2. Mendez MF, Geehan GR Jr. Cortical auditory disorders: clinical and psychoacoustic features. J Neurol Neurosurg Psychiatry 1988; 51: 1-9

3. Zatorre RJ, Evans AC, Meyer E, Gjedde A. Lateralization of phonetic and pitch discrimination in speech processing. Science 1992; 256: 846-849

4. Zhang Q, Kaga K, Hayashi A. Auditory agnosia due to long-term severe hydrocephalus caused by spina bifida-specific auditory pathway versus nonspecific auditory pathway. Acta Otolaryngologica 2011; 131: 787-792

5. Clarke S, Bellmann A, De Ribaupierre F, Assal G. Nonverbal auditory recognition in normal subjects and brain-damaged patients: evidence for parallel processing. Neuropsychologia 1996; 34: 587-603

6. Slevc LR, Marin RC, Hamilton AC, Joanisse MF. Speech perception, rapid temporal processing, and the left hemisphere: a case study of unilateral pure word deafness. Neuropsychologia 2011; 49: 216-230

7. Kitayama I, Yamazaki K, Shibahara K, Nomura J. Pure word deafness with possible transfer of language dominance. Jpn J Psychiatry Neurol 1990; 44: 577-584

8. Guyton AC, Hall JE. Textbook of medical physiology, 10th ed, Philadephia: WB Saunders, 2000, 608-609

9. Matthews BR, Chang CC, De May M, Engstrom J, Miller BL. Pleasurable emotional response to music: a case of neurodegenerative generalized auditory agnosia. Neurocase 2009; 15: 248-259 